\title{
Estimation of BER Bit Error Rate Using Digital Smoothing Filters
}

\author{
Jakub Peksinski", Grzegorz Zeglinski, Grzegorz Mikolajczak, Eugeniusz Kornatowski \\ Faculty of Electrical Engineering, West Pomeranian University of Technology, \\ Sikorskiego St. 37, 70-313 Szczecin, Poland \\ jpeksinski@zut.edu.pl
}

\begin{abstract}
In the article, the authors present a new method of numerical determination of $Q$ and BER parameters based on which the quality of transmission in optical fibre transmission systems is determined. The proposed method is based on the use of digital smoothing filters algorithms and does not require the knowledge of the so-called "eye diagram", which greatly simplifies the entire measurement procedure. This method can be implemented in a simple manner in realtime systems, and in particular it is crucial for ICT systems and networks with a high bit rate of more than $10 \mathrm{Gbps}$ and 40 Gbps and systems with time division or wavelength division multiplexing of $100 \mathrm{GBE}$ and $400 \mathrm{GBE}$.
\end{abstract}

Index Terms-Estimation; Quality index Q; Bit Error Rate; Smoothing filter.

\section{INTRODUCTION}

In every telecommunication system for data transmission, including optical transmission, the problem of signal attenuation or its distortion occurs, resulting from the physical properties of the transmission medium. At the same time, the transmitted signal between the transmitter and the receiver may be overlapped by interference (noise) generated by physical phenomena occurring in the transmission channel [1], [2]. These signals are of a random nature and are the subject of research in the field of stochastic processes theory.

The optical fibre signal transmission path - apart from the optical fibre cable - contains a number of optoelectronic devices that introduce transmission interference. Phenomena affecting the quality of the received signal include [1]-[37]:

Interference caused by the transmitter module, in particular:

- Semiconductor laser noise (phase, intensity, and mode);

- Finite spectral linewidth of semiconductor laser radiation;

- Noise and instability of generating systems.

Interference from optical fibre cables:

- Attenuation;

- Time broadening of the pulse (dispersion).

Interference generated in the receiving module:

- Detector noise (shot and thermal);

- Frequency response of the detection system.

Verification of transmission quality in radio over fibre

Manuscript received 24 February, 2021; accepted 9 August, 2021. and optical fibre systems, in accordance with the recommendations of the International Telecommunication Union - Telecommunication Standardization Sector ITU-T and International Telecommunication Union [4] Radiocommunication Sector ITU-R [5], is based on the analysis of the "eye diagram" and the determination of two important classification parameters: Quality $(Q)$ and Bit Error Rate (BER) [6], [7].

The basic criterion for assessing the quality of transmission is the characteristics of the bit error rate BER. This factor takes into account the effect of all elements of the optical fibre path and transmission system on its quality, i.e., differentiation in the receiving part of the signal sent by the transmitter, i.e., the possibility of the so-called "eye diagram mask": hexagonal or rectangular. BER measurements are used to evaluate the quality of the operation of a telecommunications system during the acceptance tests and normal operation.

One of the methods of estimating BER is the analysis of the so-called "eye diagram". It can be used to obtain a significant amount of information enabling the determination of the quality of digital signal transmission in an optoelectronic system. This diagram is obtained by overlapping all possible bit data combinations, then determining the Q parameter and estimating the BER [3], [4], [7].

A typical interpretation of the selected parameters on an eye diagram is presented in Fig. 1 .

Figure 1 shows some of the more important parameters of the eye diagram: minimum and maximum voltage values for high and low state levels $\left(\mathrm{V}_{\min }, \mathrm{V}_{\max }, \mathrm{V}^{\prime}{ }_{\text {min }}\right.$, and $\left.\mathrm{V}^{\prime}{ }_{\max }\right)$, determining mean levels of voltage interference in these states and amplitude distortion, signal distortion when switching from high to low state and from low to high state, margins determining the decision level whether a signal is a logical zero or a logical one and sampling interval.

In [35], a new accurately statistical method is proposed to estimate the worst-case eye diagrams and bit-error-rate eye diagrams for communication systems applications. The probability distribution of the coded bus bit vectors is modeled based on the coding rule constraints.

The paper [36] described a special optimization method to find the bit patterns causing the lowest received high symbol and the highest received low symbol at the sampling time point. The proposed approach is based on a mapping 
method and Bayesian optimization for solving non-linear and non-convex distortion problems, which provides a significant speedup compared to the traditional transient eye.

The article [37] proposes eye diagram estimation methods in pseudorandom binary sequence (PRBS) test and scrambling.

A more accurate method is a real-time probabilistic analysis. However, the analysis of the bit error rate in an actual system at the level of $10^{-12} \ldots 10^{-15}$ would take more than a year or several years, while the protocols of the measurement points should be implemented over a maximum of several hours to put the installation into service. ITU-T standards allow to shorten this process by the method of eye diagram analysis.
The basis for calculating the bit error rate BER is the $Q$ parameter [29]-[34]

$$
Q=\frac{\mu_{1}-\mu_{0}}{\sigma_{1}+\sigma_{0}}
$$

where $\mu_{1}$ is the mean value of the electrical signal in high state, $\mu_{o}$ is the mean value of the electrical signal in low state, $\sigma_{l}$ is the standard deviation of the electrical signal in high state, and $\sigma_{0}$ is the standard deviation of the electrical signal in low state.

Figure 2 illustrates a typical measurement eye diagram made for a temporary electrical waveform at the output of an optical fibre line.

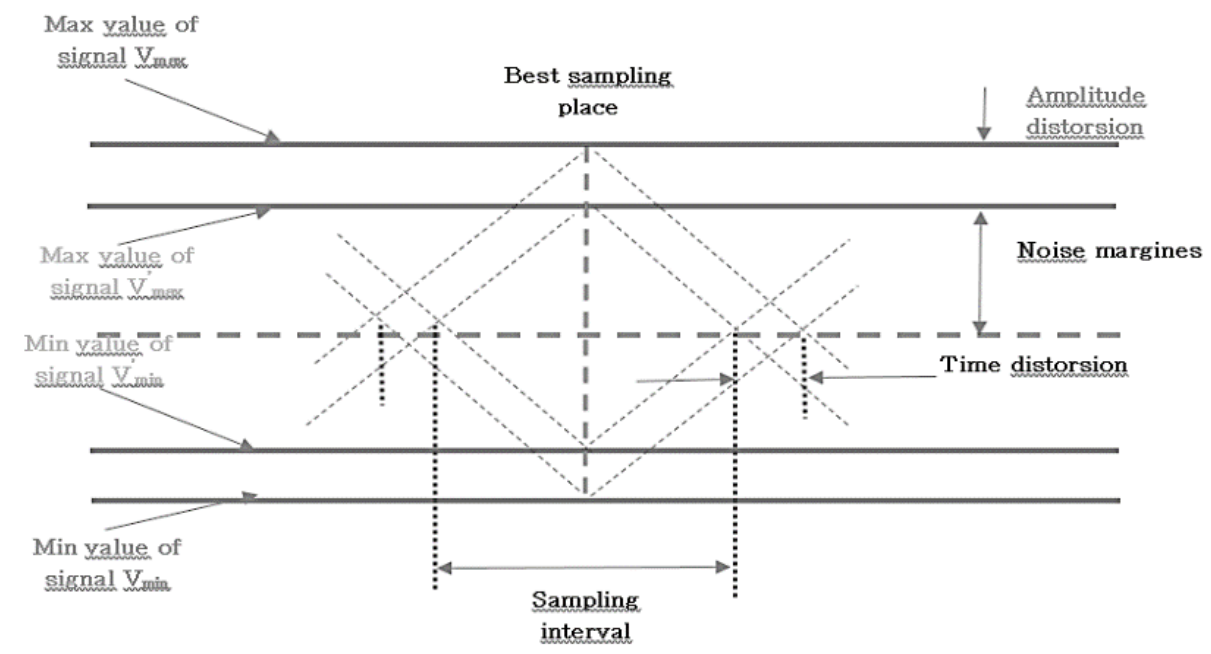

Fig. 1. Parameters of the eye diagram.

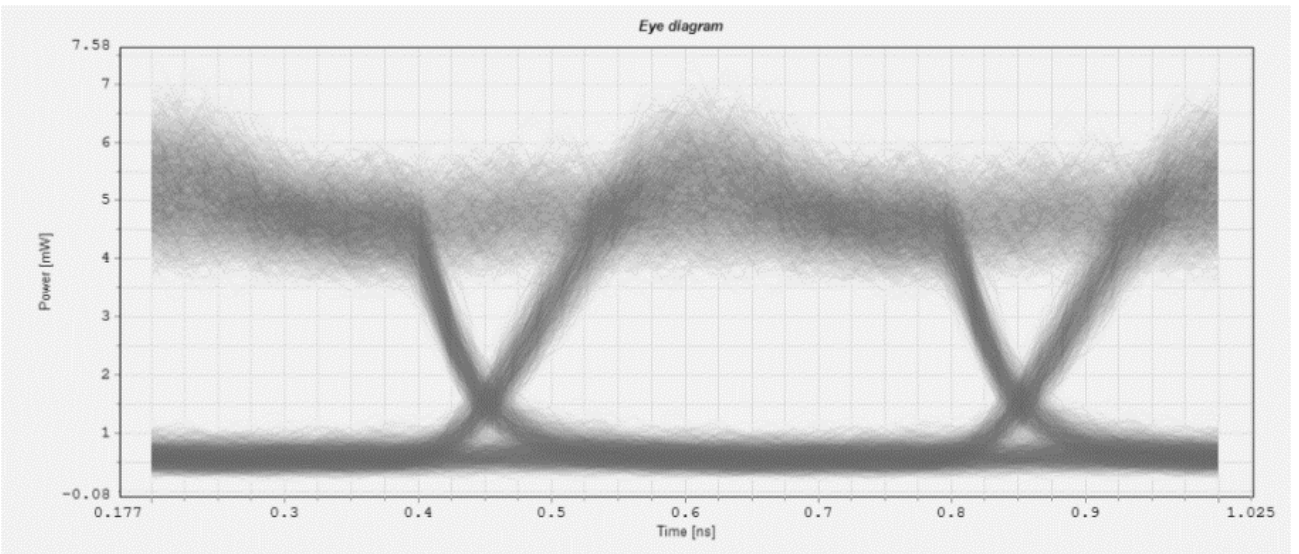

Fig. 2. Typical measurement eye diagram made for a temporary electrical waveform at the output of an optical fibre line.

The relationship between the BER and the $Q$ parameter used in telecommunications to determine the quality of the transmission is described by the relationship

$$
B E R=\frac{1}{2}\left[\operatorname{erfc}\left(\frac{Q}{\sqrt{2}}\right)\right] .
$$

The $Q$ parameter in the decibel scale is converted from the linear scale by the relationship

$$
Q_{d B}=20 \times \log (Q) \text {. }
$$

The graph showing the dependence of $Q$ parameter on the bit error rate is presented in Fig. 3.

Transmission standards in access networks require a bit error rate below the threshold BER $=10^{-12}$. It is defined in detail by the standards in [8]-[15], and the most important of them are [4], [5], [8]-[15]:

- ITU-T G.983 and ITU-T G.984 for 2.5 Gbps access networks and Ethernet networks, the criterion for bit error rate is $\mathrm{BER}<10^{-10}$,

- ITU-T G.987 (XG-PON, X-Generation Passive Optical Network -10 Gbps) and ITU-T G.989 (NG-PON2, Next 
Generation Passive Optical Network 40 Gbps), the level is BER $<10^{-12}$,

- ITU-T G.695 (CWDM, Coarse Wavelength Division Multiplexing, Nx10 Gbps), and

- ITU-T G.698 (DWDM, Dense Wavelength Division Multiplexing, terabit networks) for access and transport networks with wavelength division multiplexing, applied criterion $\mathrm{BER}<10^{-12}$.

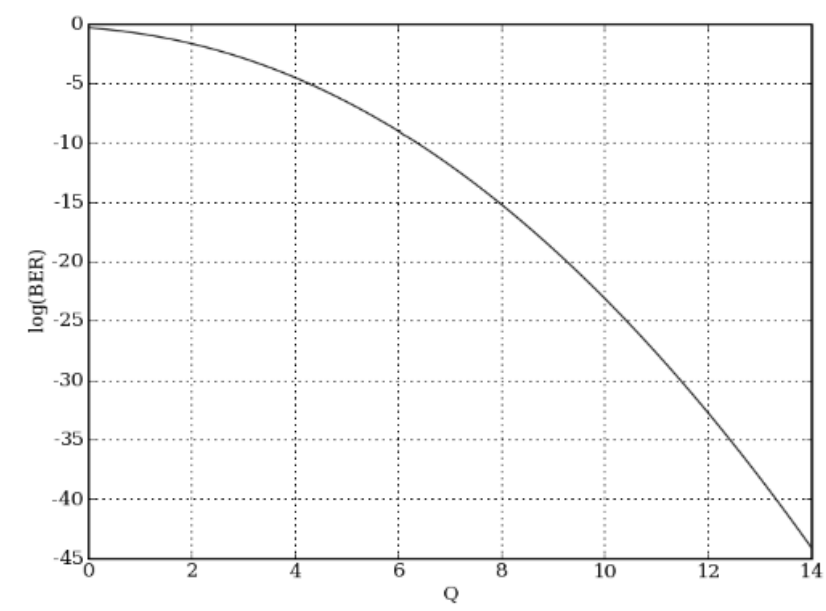

Fig. 3. The relationship of the Q parameter and the bit error rate.

The BER standard, based on the $Q$ parameter, was developed for transmissions up to $10 \mathrm{Gbps}$, and currently for transmissions with bit rate above this value, the BER values obtained with this method do not always reflect the real quality of transmission in optical networks (10 Gbps and above).

For this reason, in networks with bit rates above $10 \mathrm{Gbps}$, it is often necessary to use the observation method to estimate the quality of transmission. An expert observes the output signals or eye diagram on an ongoing basis and estimates whether the transmitted signal meets the transmission requirements.

However, this method is time-consuming and requires significant experience on the part of the individual observing the diagrams. Taking into account the development of networks and increasing the bit rate in the transmission channel, new transmission solutions [16] and the disadvantages of the "classic" use of the Q index to determine the bit error rate, this paper proposes a new, objective numerical method for estimating the value of the $\mathrm{Q}$ parameter with the use of digital smoothing filters.

\section{Q PARAMETER ESTIMATION USING DigitAl SMOOTHING FILTERS}

The value of a single sample of the observed process $x[n]$ may result from the deterministic signal model or from its statistical features that can be described by Probability Density Function (PDF). For the assumed probability distribution type, to define it unambiguously, it is enough to determine the mean value $\mu$ and the standard deviation $\sigma$ (or variance $\sigma^{2}$ ). In practice, it is unlikely that the exact values of the distribution parameters are known. For this reason, it is necessary to estimate them on the basis of the values of the observed process. In many cases, this is a difficult task, therefore the estimation process is simplified by assuming that the process is stationary and also ergodic. Adopting these assumptions enables the determination of PDF parameters solely on the basis of one observed process realization [17].

The stationary stochastic process is ergodic if, when determining any statistical characteristics, averaging over the set of realizations can be replaced by the averaging over time. In such a case, the expected value is equal to the fixed component of the selected realization, while the variance represents the average power of the realization.

These findings are the basis for estimation of the $Q$ parameter on the basis of the statistical properties of the signal (4), assuming that it is the realization of a certain stochastic process, and the observed samples are random variables of this process such that

$$
X_{k}=S_{k}+N_{k}
$$

where $S_{k}$ is the useful signal at the input, $X_{k}$ is the useful signal at the output, $N_{k}$ is the additive interference with normal distribution $N\left(0, \sigma_{n}\right)$, the average value of zero $E(N)$ $=0$, and variance $\operatorname{Var}(N)=\sigma_{n}^{2}$.

The authors of the article suggest that the $\mathrm{Q}$ parameter should be estimated by means of the following relationship

$$
Q_{\text {est }}=\frac{1}{p} \times \frac{E\left(X_{H i g h}\right)-E\left(X_{\text {Low }}\right)}{\sqrt{\operatorname{Var}\left(N_{\text {High }}\right)}+\sqrt{\operatorname{Var}\left(N_{\text {Low }}\right)}},
$$

where $E\left(X_{H i g h}\right)$ is the mean value of high signal level, $E\left(X_{\text {Low }}\right)$ is the mean value of low signal level, $\operatorname{Var}\left(N_{H i g h}\right)$ is the interference variance $N$ in high state, $\operatorname{Var}\left(N_{\text {Low }}\right)$ is the interference variance $N$ in low state, and $p$ is the correction coefficient.

In (5), there is the interference variance $\left(\operatorname{Var}(N)={\sigma_{n}}^{2}\right)$, which is to be estimated.

Methods based on signal smoothing [21]-[27] are proposed for noise variance estimation. This means that the elements of a series (data set) with an irregular diagram will be replaced by a set with a smoother diagram with suppressed interference. In the literature, such methods are also referred to as signal averaging or averaging (smoothing) filters.

Assuming that the observed signal is in the form of (4), the variance determined on the basis of the signal samples is [17]

$$
\operatorname{Var}(X)=\operatorname{Var}(S)+\operatorname{Var}(S)+2 \times \operatorname{cov}(S, N),
$$

where $\operatorname{cov}(S, N)$ is the covariance of random variables $S$ and $N$.

Assuming that the random variables $S$ and $N$ are stochastically independent (not correlated), then $\operatorname{cov}(S, N)=$ 0 and further

$$
\operatorname{Var}(X)=\operatorname{Var}(S)+\operatorname{Var}(S)
$$

To determine the interference variance, signal (4) must be filtered. For this purpose, an exponential smoothing filter was selected, described by the relationship [18] 


$$
y_{k}=\alpha \times x_{k}+(1-\alpha) \times y_{k-1}, \quad k=1 \ldots K,
$$

where $x_{k}$ and $y_{k}$ are the elements of the input and output data sets, respectively, and $y_{0}=x_{0}$. This means that the initial evaluation of the signal is the value of the chronologically earliest observation $\mathrm{x}_{\mathrm{n}}$. Parameter $\alpha \in(0,1)$ is called the "smoothing constant".

The reduction of noise variance in the case of exponential smoothing for random interference $\left\{n_{k}\right\}$ of the average value of zero $E(n)=0$ and variance $V(n)=\sigma_{n}^{2}$, is expressed by the relationship

$$
\sigma_{y}^{2}=\sigma_{n}^{2} \times \frac{\alpha}{2-\alpha}
$$

where $\sigma_{n}^{2}$ is the input noise variance, $\sigma_{y}^{2}$ is the noise variance after smoothing, and $q=\alpha /(2-\alpha)$ is the noise suppression factor.

It is a type of digital filter where the $y_{k}$ signal evaluation can be presented as a weighted average of the values of the signal from the previous period $y_{k-1}$ and the latest observation $x_{k}$

Assuming that the filtration process affects only the random variable of interference $N$, the new random variable $Y$ takes the following form

$$
Y_{k}=S_{k}+q \times N_{k}
$$

The choice of this filter was dictated by the fact that the assumption concerning reduction of just the interference is difficult to fulfil since all methods of smoothing in the frequency domain have low-pass characteristics, and thus also attenuate the useful signal [18]-[20]. For this reason, $\alpha$ $=0.95$ (smoothing factor) was used in the calculations, which caused the filter to interfere only slightly with the processed signal.

The variance of random variable $Y$ is expressed by the relationship

$$
\operatorname{Var}(Y)=\operatorname{Var}(S)+q \times \operatorname{Var}(N),
$$

where $q$ is the noise suppression factor determined analytically for the given smoothing filter.

Subtracting the sides of equations (7) and (11) and transforming, we finally obtain the relationship for the variance of the interference of the form (1), which was used to determine the values of $\operatorname{Var}\left(N_{\text {High }}\right)$ and $\operatorname{Var}\left(N_{\text {Low }}\right)$ in (5)

$$
\operatorname{Var}(N)=\frac{1}{1-q} \times[\operatorname{Var}(X)-\operatorname{Var}(Y)]
$$

The method of determining the variance of interference (12) proposed by the authors is very simple to calculate as it requires only the determination of the variance of the interfered signal and the variance of the smoothed signal $Y$, as well as the knowledge of the noise suppression coefficient $q$ - no specialist equipment is required for its determination.

Ultimately, the estimated value $Q_{e s t}$ was calculated on the basis of the relationship

$$
Q_{\text {est }}=\frac{1}{6} \times \frac{E\left(X_{\text {High }}\right)-E\left(X_{\text {Low }}\right)}{\sqrt{\operatorname{Var}\left(N_{H i g h}\right)}+\sqrt{\operatorname{Var}\left(N_{\text {Low }}\right)}},
$$

where correction parameter $p=6$.

The value of this coefficient results from the fact that the calculated $\operatorname{Var}(N)$ value represents the average power of interference, whereas $\sigma$ in (1) is a measure of the dispersion from the mean value of the signal. The assumption of $p=6$ is due to the fact that practically all values of the random variable $N$ are within the range of six standard deviations from the mean value of normal distribution $\left(\sigma \sim 6 \cdot \operatorname{Var}(N)^{1 / 2}\right)$.

\section{TeSt RESUlts}

To verify the effectiveness of the method proposed by the authors of the article for determining the $\mathrm{Q}$ parameter of optical fibre transmission, a number of tests were carried out. The tests were conducted in the Laboratory of Teleinformatic Technologies and Photonics of the West Pomeranian University of Technology in Szczecin (see Fig. 4).

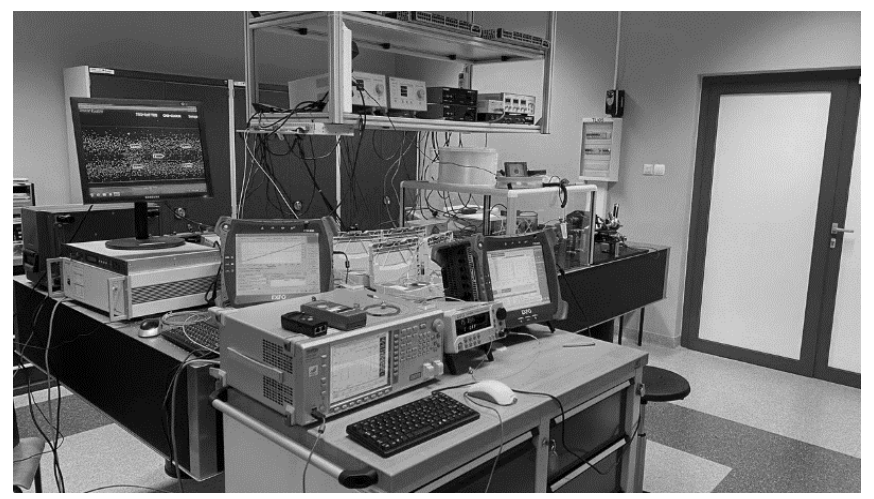

Fig. 4. Photograph of the laboratory workstation.

The first tests to evaluate and verify the method were performed in a $1 \mathrm{Gbp}$ network in the Fibre To The Home Passive Optical Network (FTTH-PON) system and were described in article [28].

The studies for $10 \mathrm{Gbps}$ bit rate were conducted in a unique Alcatel-Lucent Dense Wavelength Division Multiplexing (DWDM) terabit transport network operating in C (1530 nm-1565 nm) and L (1565 nm-1625 nm) bands with the single-channel bit rate of $10 \mathrm{Gbps}$.

The results for $40 \mathrm{Gbps}$ bit rate of the $10 \mathrm{GHz}, 40 \mathrm{GHz}$, and $100 \mathrm{GHz}$ laser linewidths of the transmitter were obtained using the eye diagram analysis method based on the nonlinear Schrödinger equation (NLSE) system using licensed RSOFT Optsim software (numerical certificate up to $160 \mathrm{Gbps}$ per channel - 2010) and VPI Software (numerical certificate 2010-2015 and 2019-2021). The software listed above enables the analysis of transmissions above 1 Tbp.

The existing Alcatel-Lucent and Orange systems have numerical and hardware certificates for $10 \mathrm{Gbps}$ singlechannel transmission in the $\mathrm{C}(1530 \mathrm{~nm}-1565 \mathrm{~nm})$ an $\mathrm{L}$ $(1565 \mathrm{~nm}-1625 \mathrm{~nm})$ band, as well as support on three software levels.

The system was designed with RSOFT software and then verified with Alcatel-Lucent's service software. The licence 
enables generating the bit rate of $1 \mathrm{Tbp}$ in the $\mathrm{C}$ band, and in the case of Orange - $800 \mathrm{Gbps}$ in the $\mathrm{C}$ and L bands. The certificate allows to expand the equipment and software up to the bit rate of $10 \mathrm{Tbps}$.

The measuring equipment owned and used by the laboratory comes from and has certificates of a recognised EXFO company in the field of power measurement devices, Optical Time Domain Reflectometer (OTDR), optical spectrum analysis, chromatic and polarization mode dispersion; the eye diagrams are prepared on equipment certified by Alnair Labs (optical oscilloscope operating in the $\mathrm{C}$ band), eye diagram analysis for any None Return to Zero (NRZ) and Return to Zero (RZ) codes, (band-width
$500 \mathrm{GHz}, 640 \mathrm{Gbps}$ in a single channel), and Tektronix equipment (eye diagram analysis up to $2.5 \mathrm{GHz}$ ) (see Fig. 5).

The systems use certified components from Thorlabs, FCA, and Hewlett Packard.

A reference test signal (see Fig. 6) with $N=8192$ samples and the following parameters were used for the tests:

$-\mathrm{Q} \sim 30 \mathrm{~dB}$

$-\mu 1 \sim 2.5 \times 10^{-5}$ - mean value of the signal in high state;

$-\mu \mathrm{o} \sim 2.5 \times 10^{-6}$ - mean value of the signal in low state.

The time diagram of the reference test signal is presented in Fig. 6.

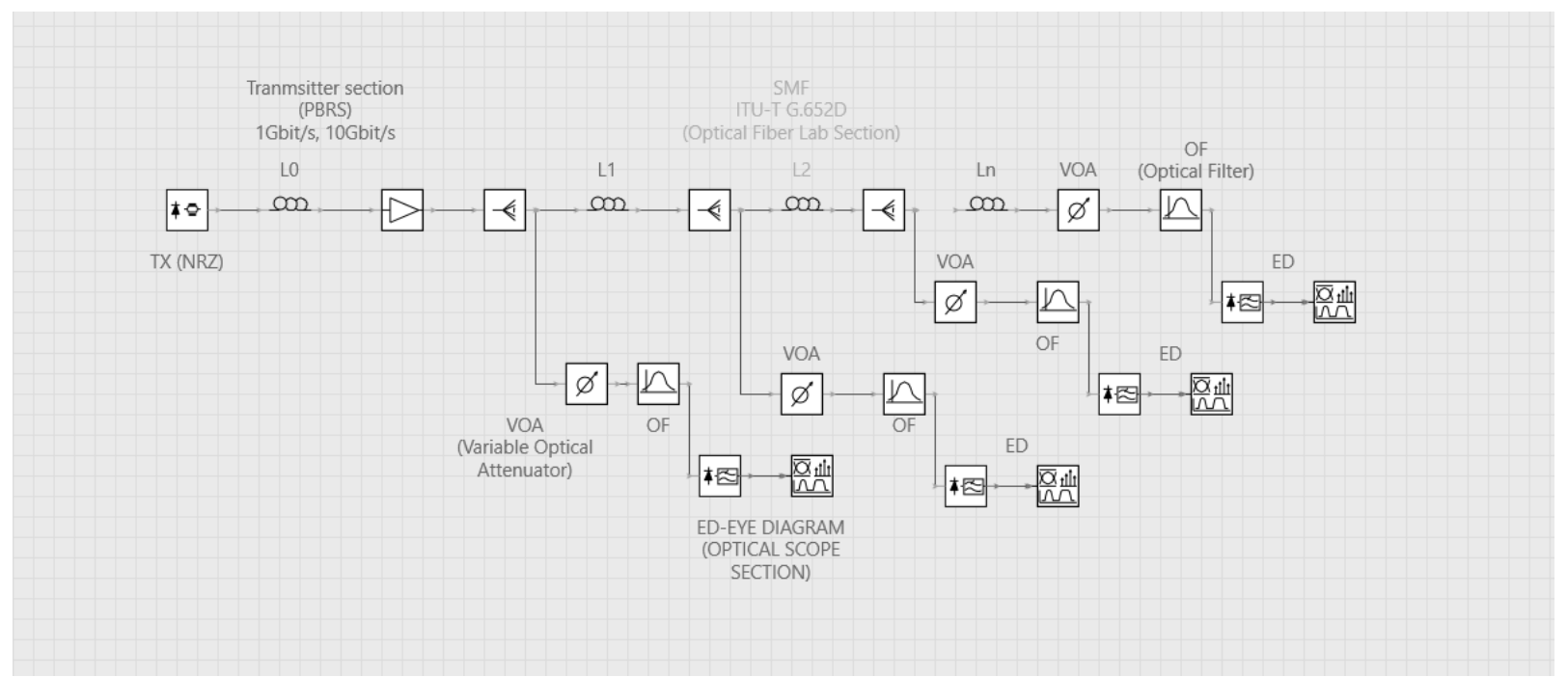

Fig. 5. Schematic diagram of the measuring circuit.

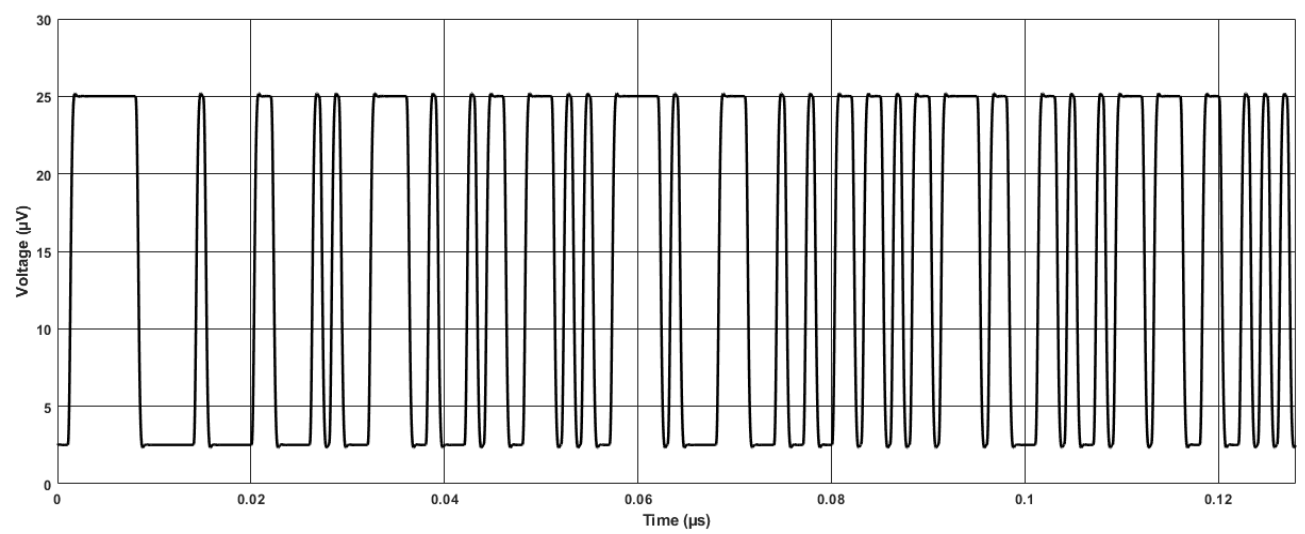

Fig. 6. Reference signal at transmitter output, $\mathrm{Q} \sim 30 \mathrm{~dB}, \mu 1 \sim 2.5 \times 10^{-5}$ - mean value of the signal in high state, $\mu \mathrm{o} \sim 2.5 \times 10^{-6}$ - mean value of the signal in low state.

To verify the effectiveness of the method proposed by the authors, a number of experiments were carried out.

The first experiment consisted of an artificial distortion of the reference signal with noise $\left\{n_{k}\right\}$, with normal distribution (Gauss), average value of zero $E(n)=0$, and variance $V(n)$ $=\sigma_{n}^{2}$, where the standard deviation value $\sigma_{n}$ changed in the range of $\sigma_{n}=1.0 \times 10^{-6}-2.0 \times 10^{-6}$, which corresponds to $\mathrm{Q}$ $=20 \mathrm{~dB}-14 \mathrm{~dB}$.

Then the value of $Q_{\text {est }}$ was estimated using (13). The results of the experiment are presented in Table I.

The experiment confirmed the effectiveness of the proposed method in the range of the assumed interference level. The results shown in Table I are highly consistent with the setpoint parameters of interference. Relative error value $\mathrm{Q}_{\text {est }}$ did not exceed $1.4 \%$ and had a decreasing tendency. The lowest value $(0.338 \%)$ was observed for $\sigma=$ $2,0 \times 10^{-6}$, i.e., at a relatively high level of interference.

The second experiment was based on the estimation of the $Q$ parameter value from the output signals obtained for $10 \mathrm{Gbps}$ transmission and $10 \mathrm{GHz}$ laser linewidth for sections with the length of $10 \mathrm{~km}, 15 \mathrm{~km}, \ldots, 35 \mathrm{~km}$.

The third experiment was based on the estimation of the $Q$ parameter value from the output signals obtained for $40 \mathrm{Gbps}$ transmission and $10 \mathrm{GHz}, 40 \mathrm{GHz}$, and $50 \mathrm{GHz}$ 
laser linewidths for sections with the length of from $10 \mathrm{~m}$ to $1600 \mathrm{~m}$.

The obtained results are presented in Tables II and III. Examples of the time diagrams of the output signals are shown in Figs. 7-9. As can be noted, the individual signals are attenuated and increasingly distorted.

The results of the studies presented in Tables II and III indicate that the proposed algorithm is highly effective. It is especially visible in the case when the classic (described in the standards) method of analysing the eye diagram indicates the value of BER significantly deviating from the norm for transmission at a given bit rate due to problems with mask application (Fig. 10 - closed eye). In this case, the expert can estimate the quality of the output signal.

TABLE I. RESULTS OF THE EXPERIMENT CONSISTING IN THE ESTIMATION OF $\sigma$ ( $\sigma 2$ - NOISE POWER) AND Q FROM THE REFERENCE SIGNAL, PRESENTED IN FIG. 6, DISTURBED BY NOISE OF NORMAL DISTRIBUTION N $(0, \sigma)$,WHERE $\sigma=1.0 \times 10^{-6}-2.0 \times 10^{-6}$, WHICH CORRESPONDS TO $\mathrm{Q}=20 \mathrm{~dB}-14 \mathrm{~dB}$.

\begin{tabular}{|c|c|c|c|c|c|c|c|}
\hline No. & $\begin{array}{c}\sigma \text { - set point } \\
\text { value }\end{array}$ & $Q[d B]$ & BER & $\begin{array}{l}\text { Estimated } \\
\text { value of } \sigma\end{array}$ & $Q_{\text {est }}[\mathrm{dB}]$ & $\begin{array}{c}\text { BER calculated } \\
\text { from } Q_{\text {est }} \\
\text { estimated }\end{array}$ & $\begin{array}{l}\text { Relative error in } \\
\% \text { of } Q \text { estimate } \\
\text { in } \mathrm{dB}\end{array}$ \\
\hline 1 & $1.0 \times 10^{-6}$ & 20.32 & $1.61 \cdot \times 10^{-25}$ & $1.033 \cdot \times 10^{-6}$ & 20.044 & $4.56 \cdot \times 10^{-24}$ & 1.378 \\
\hline 2 & $1.1 \times \cdot 10^{-6}$ & 19.49 & $2.10 \times \cdot 10^{-21}$ & $1.129 \times 10^{-6}$ & 19.269 & $1.91 \cdot \times 10^{-20}$ & 1.169 \\
\hline 3 & $1.2 \times \cdot 10^{-6}$ & 18.74 & $2.58 \cdot \times 10^{-18}$ & $1.226 \times 10^{-6}$ & 18.553 & $1.28 \times \cdot 10^{-17}$ & 1.001 \\
\hline 4 & $1.3 \times \cdot 10^{-6}$ & 18.04 & $7.32 \cdot \times 10^{-16}$ & $1.324 \cdot \times 10^{-6}$ & 17.889 & $2.21 \times \cdot 10^{-15}$ & 0.865 \\
\hline 5 & $1.4 \times 10^{-6}$ & 17.40 & $6.17 \cdot \times 10^{-14}$ & $1.422 \cdot \times 10^{-6}$ & 17.271 & $1.40 \times \cdot 10^{-13}$ & 0.752 \\
\hline 6 & $1.5 \times \cdot 10^{-6}$ & 16.80 & $2.28 \times 10^{-12}$ & $1.520 \cdot \times 10^{-6}$ & 16.692 & $4.16 \cdot \times 10^{-12}$ & 0.656 \\
\hline 7 & $1.6 \cdot \times 10^{-6}$ & 16.24 & $4.40 \cdot \times 10^{-11}$ & $1.618 \times 10^{-6}$ & 16.149 & $6.87 \cdot \times 10^{-11}$ & 0.575 \\
\hline 8 & $1.7 \times 10^{-6}$ & 15.72 & $4.99 \cdot \times 10^{-10}$ & $1.716 \times 10^{-6}$ & 15.636 & $7.21 \cdot \times 10^{-10}$ & 0.504 \\
\hline 9 & $1.8 \times 10^{-6}$ & 15.22 & $4.02 \cdot \times 10^{-9}$ & $1.815 \times 10^{-6}$ & 15.152 & $5.24 \times 10^{-9}$ & 0.442 \\
\hline 10 & $1.9 \cdot \times 10^{-6}$ & 14.75 & $2.33 \cdot \times 10^{-8}$ & $1.914 \times 10^{-6}$ & 14.692 & $2.86 \cdot \times 10^{-8}$ & 0.387 \\
\hline 11 & $2.0 \times 10^{-6}$ & 14.30 & $1.06 \cdot \times 10^{-7}$ & $2.013 \times \cdot 10^{-6}$ & 14.256 & $1.22 \cdot \times 10^{-7}$ & 0.338 \\
\hline
\end{tabular}

TABLE II. RESULTS FOR 10 GBPS BIT RATE

\begin{tabular}{|c|c|c|c|c|c|c|}
\hline $\begin{array}{c}\text { Transmission } \\
\text { distance } \\
{[\mathbf{m}]}\end{array}$ & $\begin{array}{c}\text { Transmitter } \\
\text { laser spectrum } \\
\text { linewidth } \\
{[\mathbf{G H z}]}\end{array}$ & Q parameter & BER & $\begin{array}{c}\text { Expert } \\
\text { Evaluation }\end{array}$ & $\begin{array}{c}\text { BER } \\
\text { Qest } \\
\text { Estimated }\end{array}$ & $\begin{array}{c}\text { Calculated from } \\
\text { Q estimated }\end{array}$ \\
\hline 10 & 5 & 21.17 & 0.00 & 1 & 15.01 & $5.3 \times 10^{-51}$ \\
\hline 100 & 5 & 13.70 & $3.8 \times 10^{-41}$ & 1 & 12.32 & $5.5 \times 10^{-35}$ \\
\hline 500 & 5 & 10.27 & $3.5 \times 10^{-25}$ & 2 & 11.30 & $7.0 \times 10^{-30}$ \\
\hline 700 & 5 & 7.14 & $3.8 \times 10^{-13}$ & 2 & 10.34 & $3.1 \times 10^{-24}$ \\
\hline 900 & 5 & 3.76 & $8.1 \times 10^{-5}$ & 3 & 8.22 & $1.2 \times 10^{-16}$ \\
\hline 1100 & 5 & 2.73 & 0.0031 & 3 & 5.61 & $1.1 \times 10^{-8}$ \\
\hline
\end{tabular}

Note: where expert evaluation: 1 - Transmission quality very good, 2 - ITU-T-compliant quality, 3 - Laboratory quality (below standards, but correction of errors possible).

TABLE III. RESULTS FOR 40 GBPS BIT RATE.

\begin{tabular}{|c|c|c|c|c|c|c|}
\hline $\begin{array}{c}\text { Transmission } \\
\text { distance } \\
{[\mathbf{m}]}\end{array}$ & $\begin{array}{c}\text { Transmitter } \\
\text { laser spectrum } \\
\text { linewidth } \\
{[\mathbf{G H z}]}\end{array}$ & Q parameter & BER & $\begin{array}{c}\text { Expert } \\
\text { Evaluation }\end{array}$ & $\begin{array}{c}\text { Qest } \\
\text { Estimated }\end{array}$ & $\begin{array}{c}\text { BER } \\
\text { Calculated from } \\
\text { Q estimated }\end{array}$ \\
\hline 10 & 10 & 52.59 & 0.00 & 1 & 29.10 & $4.4 \times 10^{-179}$ \\
\hline 100 & 10 & 25.79 & 0.00 & 1 & 22.70 & $3.0 \times 10^{-114}$ \\
\hline 500 & 10 & 11.10 & $4.6 \times 10^{-29}$ & 2 & 20.73 & $9.7 \times 10^{-96}$ \\
\hline 700 & 10 & 7.50 & $2.3 \times 10^{-14}$ & 2 & 15.99 & $6.8 \times 10^{-58}$ \\
\hline 900 & 10 & 6.15 & $2.7 \times 10^{-10}$ & 2 & 15.26 & $7.4 \times 10^{-53}$ \\
\hline 1100 & 10 & 5.11 & $1.3 \times 10^{-7}$ & 3 & 13.55 & $3.9 \times 10^{-42}$ \\
\hline 1600 & 10 & 4.10 & $1.6 \times 10^{-5}$ & 3 & 11.24 & $1.3 \times 10^{-29}$ \\
\hline 200 & 50 & 11.74 & $3.0 \times 10^{-32}$ & 2 & 21.95 & $4.5 \times 10^{-107}$ \\
\hline 400 & 50 & 5.67 & $5.6 \times 10^{-9}$ & 2 & 16.51 & $2.0 \times 10^{-61}$ \\
\hline 600 & 50 & 4.20 & $1.1 \times 10^{-5}$ & 3 & 8,712 & $1.1 \times 10^{-16}$ \\
\hline 800 & 50 & 3.37 & $3.1 \times 10^{-4}$ & 3 & 3.12 & $9.1 \times 10^{-4}$ \\
\hline 200 & 100 & 8.14 & $1.7 \times 10^{-16}$ & 2 & 35.51 & 0.00 \\
\hline 400 & 100 & 4.07 & $1.9 \times 10^{-5}$ & 3 & 6.41 & $7.3 \times 10^{-11}$ \\
\hline 600 & 100 & 3.08 & $8.8 \times 10^{-4}$ & 3 & & 4.53 \\
\hline 800 & 100 & 2.23 & 0.0125 & No eye diagram & 3.43 & $2.9 \times 10^{-6}$ \\
\hline
\end{tabular}

Note: where expert evaluation: 1 - Transmission quality very good, 2 - ITU-T-compliant quality, 3 - Laboratory quality (below standards, but correction of errors possible). 


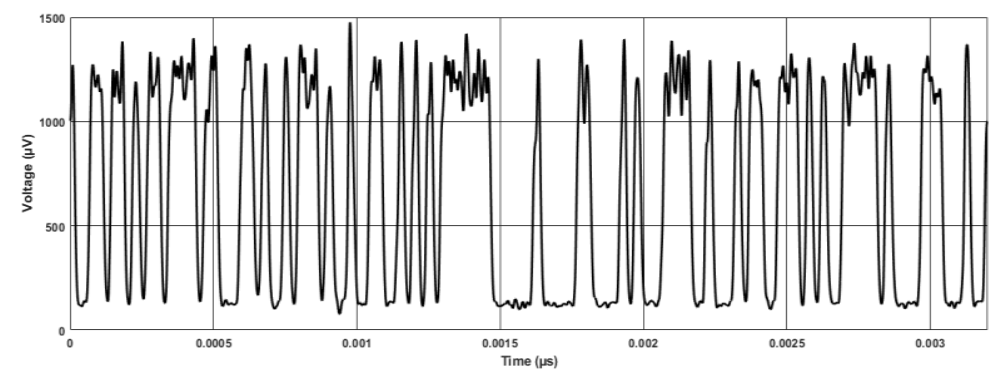

Fig. 7. Signal at detector output - optical fibre cable length $\mathrm{L}=500 \mathrm{~m}$, bit rate $40 \mathrm{Gbps}$ and spectrum linewidth $10 \mathrm{Ghz}$, $\mathrm{Q} \sim 11 \mu 1 \sim 1,2 \cdot \times 10^{-3}-$ mean value of the signal in high state, $\mu \mathrm{o} \sim 1.2 \times 10^{-4}$ - mean value of the signal in low state reference signal at transmitter output, $\mathrm{Q} \sim 30 \mathrm{~dB}, \mu 1 \sim 2.5 \cdot \times 10^{-5}-$ mean value of the signal in high state, $\mu \mathrm{o} \sim 2.5 \times 10^{-6}$ - mean value of the signal in low state.

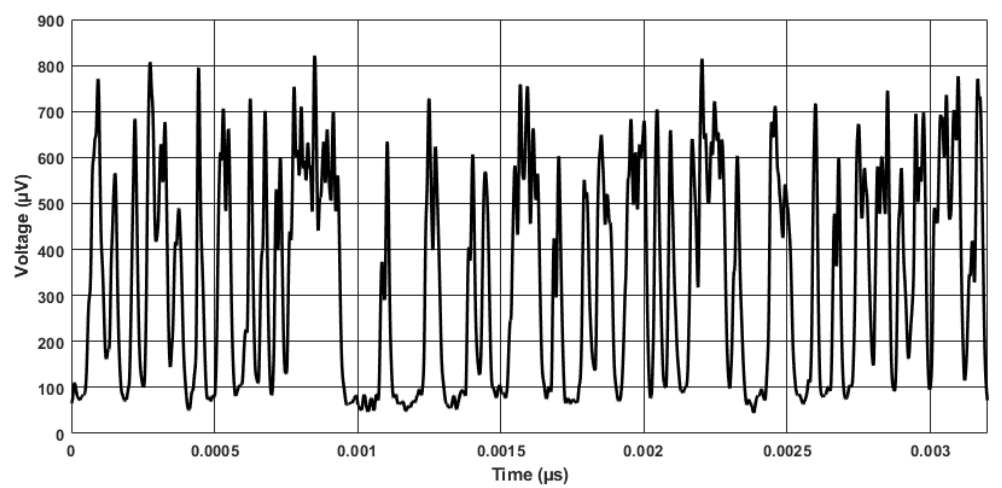

Fig. 8. Signal at detector output - optical fibre cable length $\mathrm{L}=400 \mathrm{~m}$, bit rate $40 \mathrm{Gbps}$ and spectrum linewidth $100 \mathrm{Ghz}, \mathrm{Q} \sim 4.1 \mu 1 \sim 6 \times 10^{-4}$ - mean value of the signal in high state, $\mu_{\mathrm{o}} \sim 1 \times 10^{-4}$ - mean value of the signal in low state.

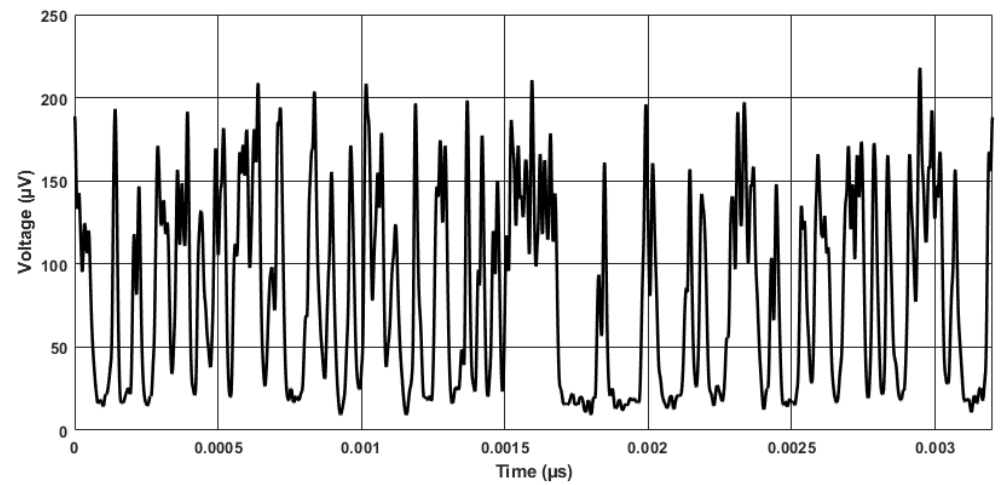

Fig. 9. Signal at detector output - optical fibre cable length $\mathrm{L}=500 \mathrm{~m}$, bit rate $40 \mathrm{Gbps}$ and spectrum linewidth $50 \mathrm{Ghz}, \mathrm{Q} \sim 5.7 \mu 1 \sim 1.4 \times 10^{-4}-\mathrm{mean}$ value of the signal in high state, $\mu \mathrm{o} \sim 2 \times 10^{-5}$ - mean value of the signal in low state.

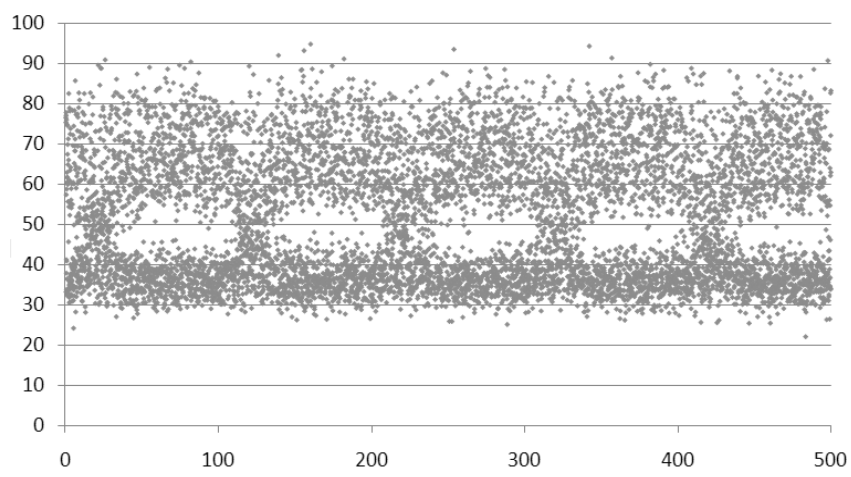

Fig. 10. Actual eye diagram for the Alcatel-Lucent system $(B=10 \mathrm{Gbps}$ $\mathrm{FWHM}=10 \mathrm{GHz}, 35 \mathrm{~km})-$ critical case BER $=0.0031($ copy of the oscilloscope screen).

The intervention of an expert is unnecessary, which greatly simplifies and speeds up the measurement procedure.

It is also possible to recover the parameters of the eye diagram by means of forward error correction (FEC) and determine whether the signal is suitable for transmission despite significant interference after error correction - socalled "laboratory quality". In this case, the BER parameter, determined by the algorithm presented by the authors, unambiguously defines the quality of transmission as satisfactory.

The results of the experiments collected in the tables show that the method proposed in this paper works in each case. It is worth noting that the method is also effective in cases of a critical decrease of the transmission distance resulting from high bit rate.

\section{CONCLUSIONS}

The proposed method has been evaluated in the Laboratory of Teleinformatic Technologies and Photonics of the West Pomeranian University of Technology in Szczecin. The laboratory has dedicated modern equipment 
for testing access and transport systems with $1 \mathrm{Gbp}$, $2.5 \mathrm{Gbps}$, and $10 \mathrm{Gbps}$ bit rate in a single channel and $40 \mathrm{Gbps}, 80 \mathrm{Gbps}, 400 \mathrm{Gbps}$, and $800 \mathrm{Gbps}$ in systems with multiplexing. The equipment was provided by Orange and Alcatel-Lucent. The Alcatel Lucent system can provide transmissions of 3.2 Tbps (88 channels at $40 \mathrm{Gbps}$ ).

The conducted research indicates good applicability of the existing classic algorithms of eye diagram evaluation for networks with bit rates up to $10 \mathrm{Gbps}$, and in particular:

- 1 Gbp (1 Gigabit Ethernet);

- 1.25 Gbps (GPON, Gigabit Passive Optical Network);

-2.5 Gbps (GPON);

- Up to 10 Gbps (10 Gigabit Ethernet).

While in systems with bit rates:

- Over 10 Gbps with a range exceeding $10 \mathrm{~km}-20 \mathrm{~km}$ (WDM, XG-PON);

- 40 Gbps and higher (NG-PON2, DWDM);

- There are additional interference effects in the eye diagrams which cause that the classic determination of the $\mathrm{Q}$ parameter and the following determination of the bit error rate BER may lead to incorrect estimates and conclusions. In such cases, the method based on expert observation plays an important role. However, this method is time-consuming and requires a high level of knowledge and experience.

The method proposed by the authors in this study clearly correlates with classic methods (described in standards) for bit rates up to $10 \mathrm{Gbps}$, confirming its effectiveness. Its main advantage is the unambiguous and objective result of diagnostic analysis in the case of tests at bit rates above $10 \mathrm{Gbps}$. For this range of bit rates, the proposed method provides results consistent with the expert assessment made on the basis of observation (subjective assessment) of the initial diagrams and eye diagram correction. An additional advantage of the proposed method is its simplicity - it can be implemented in real-time devices, where the measurement of the transmission parameters is performed on an ongoing basis.

\section{CONFLICTS OF INTEREST}

The authors declare that they have no conflicts of interest.

\section{REFERENCES}

[1] C. C. Davis, Lasers and Electro-optics - Fundamentals and Engineering, 2nd ed. Cambridge University Press, 2014.

[2] S. Quimby, Photonics and Lasers. New Jersey: J. Wiley \& Sons, Inc., 2006. DOI: 10.1002/0471791598.

[3] J. Siuzdak, Photonic Systems and Networks. WKL, Warszawa, 2009.

[4] ITU Telecommunication Standardization Sector, Comitted to connecting the world, ITU. [Online]. Available: https://www.itu.int/en/ITU-T/Pages/default.aspx

[5] ITU-R Recommendations, Comitted to connecting the world, ITU. [Online]. Available: https://www.itu.int/pub/R-REC

[6] B Gunther and D. Steel, Encyclopedia of Modern Optics. Elsevier Ltd., 2005.

[7] S. Stanley, "From 25/100G to 400G: A competitive analysis of optical modules \& components", Heavy Reading Report, vol. 13, no. 08, 2015.

[8] Recommendation ITU-G 983: "Broadband optical access systems based on Passive Optical Networks (PON)", 2005.

[9] Recommendation ITU-G 984: "Gigabit-capable passive optical networks (GPON): General characteristics", 2008.

[10] Recommendation ITU-G 989: "40-Gigabit-capable passive optical networks (NG-PON2): Definitions, abbreviations and acronyms", 2015.
[11] Recommendation ITU-G 695: "Optical interfaces for coarse wavelength division multiplexing applications", 2018.

[12] Recommendation ITU-G 698: "Multichannel DWDM applications with single-channel optical interface", 2009.

[13] Recommendation ITU-G 987: "10-Gigabit-capable passive optical network (XG-PON) systems: Definitions, abbreviations and acronyms", 2012.

[14] Recommendation ITU-T G.652: "Characteristic of single-mode optical fibre and cable", 06/2005.

[15] Recommendation ITU-R P.1814: "Prediction methods required for the design of terrestrial free-space optical link", 2007.

[16] R. Ramaswami, K. N. Sivarajan, and G. H. Sasaki, Optical Networks A Practical Perspective. Elsevier Inc., 2010.

[17] P. Z. Peebles, Probability Random Variables and Random Signal Principles, 4th ed. Boston: McGraw-Hill, 2001.

[18] R. G. Lyons, Understanding Digital Signal Processing, 3rd ed. Prentice Hall, 2010.

[19] D. Schlichthärle, Digital Filters. Springer, 2011. DOI: 10.1007/978-3642-14325-0.

[20] S. Winder, Analog and Digital Filter Design. Newnes, 2002. DOI: 10.1016/B978-075067547-5/50016-6.

[21] J. P. Kowalski, J. Peksinski, and G. Mikolajczak, "Detection of noise in digital images by using the averaging filter name COV", Intelligent Information and Database Systems, ACIIDS 2013. Lecture Notes in Computer Science, vol. 7803. Springer, Berlin, Heidelberg, 2013. DOI: 10.1007/978-3-642-36543-0_1.

[22] G. Mikolajczak and J. Peksinski, "Estimation of the variance of noise in digital images using a median filter", in Proc. of 39th International Conference on Telecommunications and Signal Processing (TSP), 2016, pp. 489-492. DOI: 10.1109/TSP.2016.7760927.

[23] J. Peksinski, G. Mikolajczak, and J. P. Kowalski, "Estimation of the level of disturbance in time series using a median filter", in New Research in Multimedia and Internet Systems. Advances in Intelligent Systems and Computing, vol. 314. Springer, Cham, 2015. DOI: 10.1007/978-3-319-10383-9_9.

[24] G. Georgis, G. Lentaris, and D. Reisis, "Acceleration techniques and evaluation on multi-core CPU, GPU and FPGA for image processing and super-resolution", Journal of real-time image processing, vol. 16, no. 4, pp. 1207-1234, 2019. DOI: 10.1007/s11554-016-0619-6.

[25] J. Zhang, Y. Cao, Z.-J. Zha, Z. Zheng, C. W. Chen, and Z. Wang, “A unified scheme for super-resolution and depth estimation from asymmetric stereoscopic video", IEEE Transactions on Circuits and Systems for Video Technology, vol. 26, no. 3, pp. 479-493, 2016. DOI: 10.1109/TCSVT.2014.2367356.

[26] J. Wu, F. You, and P. Jiang, "Noise variance estimation method based on regression analysis and principal component analysis", Journal of Electronics \& Information Technology, vol. 40, no. 5, pp. 1195-1201, 2018. DOI: 10.11999/JEIT170624.

[27] N. I. Chervyakov, P. A. Lyakhov, A. R. Orazaev, and M. V. Valueva, "Efficiency analysis of the image impulse noise cleaning using median filters with weighted central element", in Proc. of 2017 International Multi-Conference on Engineering, Computer and Information Sciences (SIBIRCON), 2017, pp. 141-146. DOI: 10.1109/SIBIRCON.2017.8109856.

[28] G. Żegliński, J. Pęksiński, and G. Mikołajczak, "SNR estimation using digital filters for fiber optic lines", Poznan University of Technology Academic Journals. Electrical Engineering, vol. 97, pp. 191-202, 2019 (in polish). DOI: 10.21008/j.1897-0737.2019.97.0017.

[29] G. P. Agrawal, Fiber Optic Communication Systems. John Wiley and Sons, Inc., 2010. DOI: 10.1002/9780470918524.

[30] S. Verma, A. Kakati, M. R Tripathy, A. K. Singh, S. Kumar, and H. Rana, "Performance analysis of GPON network for maximum QFACTOR and minimum BER for various fiber length using uniform fiber Bragg's grating”, in Proc. of International Conference on Information Technology (In CiTe) - The Next Generation IT Summit on the Theme - Internet of Things: Connect your Worlds, 2016, pp. 247-250. DOI: 10.1109/INCITE.2016.7857625.

[31] I. Shake, H. Takara, and S. Kawanishi, "Simple Q factor monitoring for BER estimation using opened eye diagrams captured by highspeed asynchronous electrooptical sampling", IEEE Photonics Technology Letters, vol. 15, no. 4, pp. 620-622, Apr. 2003. DOI: 10.1109/LPT.2003.809316.

[32] T. A. Lindsay, "Innovations in BER testers enable fast and accurate eye diagram, eye mask, Q-factor, and jitter measurements", in Proc. of Optical Fiber Communication Conference, 2004. OFC 2004, 2004, p. 4, vol. 2.

[33] G. Li, J. Deng, S. Xin, and X. G. Huang, “A radio over fiber system compatible with $3 \mathrm{G} / 4 \mathrm{G} / 5 \mathrm{G}$ for full spectrum access and handover 
with multi-scenarios", Journal of Lightwave Technology (Early access), 2021. DOI: 10.1109/JLT.2021.3065568.

[34] Y. Zhang, J. Miao, Z. Zhang, M. Nie, Z. He, and W. Zhao, "Eyediagram measurement of high-speed digital signals using sampling oscilloscope with time-base correction", in Proc. of 15th IEEE International Conference on Signal Processing (ICSP), 2020, pp. 1114. DOI: $10.1109 /$ ICSP48669.2020.9321053.

[35] X. Chu, W. Wang, J. Wang, X. Dai, J. Fan, and Y. Li, "Statistical eye diagram analysis based on double-edge responses for coding buses", IEEE Transactions on Electromagnetic Compatibility, vol. 62, no. 3 , pp. 902-913, 2020. DOI: 10.1109/TEMC.2019.2923254.

[36] M. A. Dolatsara and M. Swaminathan, "Determining worst-case eye height in low BER channels using Bayesian optimization", IEEE 11th Latin American Symposium on Circuits \& Systems (LASCAS), 2020, pp. 1-4. DOI: 10.1109/LASCAS45839.2020.9069049.

[37] J. Park, S. Park, Y. Kim, G. Park, H. Park, D. Lho, K. Cho, S. Lee, D. H. Kim, and J. Kim, "Polynomial model-based eye diagram estimation methods for LFSR-based bit streams in PRBS test and scrambling", IEEE Transactions on Electromagnetic Compatibility, vol. 61, no. 6, pp. 1867-1875, 2019. DOI: 10.1109/TEMC.2019.2900055.

This article is an open access article distributed under the terms and conditions of the Creative Commons Attribution 4.0 (CC BY 4.0) license (http://creativecommons.org/licenses/by/4.0/). 